\title{
Phenomenal qualities and the development of perceptual integration
}

\author{
Mariann Hudak, ${ }^{1,2}$ Zoltan Jakab, ${ }^{2}$ Ilona Kovacs ${ }^{1,2}$ \\ ${ }^{1}$ Research Group of Cognitive Science, Hungarian Academy of Sciences - Budapest University of Technology \\ and Economics, Budapest, Hungary \\ ${ }^{2}$ Department of Cognitive Science, Budapest University of Technology and Economics, Budapest, Hungary
}

\begin{abstract}
In this chapter, data concerning the development of principal aspects of vision is reviewed. First, the development of colour vision and luminance perception is discussed. Relevant data accumulated so far indicates that perception of colour and luminance is present by 6-9 months of age. The presence of typical color illusions at this age suggests that the phenomenal character of color experience is comparable to that of adults well before the first birthday. Thus it seems plausible that color perception develops on the grounds of genetically preprogrammed maturation, in which perceptual learning and plasticity play a limited role. This claim also seems to be supported by case studies of newly sighted patients. On the other hand, perceptual integration of edges and forms in the visual environment as well as the development of binocular vision, and the perception of ambiguous figures seem to depend crucially on early perceptual learning. Both developmental and clinical data indicate a more significant involvement of perceptual learning in such mid-level and higher level perceptual phenomena.
\end{abstract}

\section{Introduction}

When investigating perceptual development, an inherently interesting question is, what is children's perceived world like, and how does it differ from that of adults? The data reviewed in this chapter is intended to shed some light on this question. Part of the question is, what can we know about children's phenomenal experiences; is their experience, in terms of its phenomenal character, similar to that of adults? Perceptual experiences have a characteristically subjective aspect, which is accessible only from the subject's first-person point of view, namely what it is like to undergo a given experience. This subjective aspect is called phenomenal character. ${ }^{1}$ In a sense, experiences are states of the brain, and they can be described as such - for example, in terms of neuroscience. But since your brain states (neuronal activities) do not, and cannot, occur in my brain, I cannot undergo your experiences. $^{2}$ This is part of the explanation of what makes experience subjective.

\footnotetext{
${ }^{1}$ In a broad sense, the terms 'qualia' and 'phenomenal character' have the same meaning: they refer to the subjective aspect of experiences (see the main text). The term 'qualia', however, is more strongly associated with a certain theoretical conception of the nature of this subjective character, namely the idea that qualia are intrinsic properties of certain brain states. Intrinsic properties are local properties that follow from the way something is mass and chemical composition are examples. Other (non-intrinsic) properties like weight or inflammability arise from how entities interact with the world. According to representational theories of phenomenal consciousness (e.g., Tye, 2000), the subjective aspect is not an intrinsic property of brain states, but rather it arises from how our brain states interact with the environment. Thus whether the subjective aspect of experiences is one of their intrinsic properties is a matter of debate. The term 'phenomenal character' is a theoretically more neutral one for the subjective aspect than 'qualia'.

${ }^{2}$ I can only study your brain activity, say, by brain imaging. But via the brain imaging equipment, I am not properly related to your brain activity to have the phenomenal experience that you have when that activity occurs in your brain. In this sense experiences are relational properties; an experience is, at a minimum, (i) a local neural activity which is (ii) properly related to the rest of one's brain. These are two general conditions for
} 
One might ask, in what sense is the question about children's phenomenal experiences different from the more general one about their perceived world? After all, perceptual integration plays a key role in bringing about the subjective aspect of experience. Children do not experience certain illusions the same way as adults do - to them, the same stimuli look subjectively different than to adults (and to some extent they can behaviourally indicate their way of experiencing those stimuli).

It is also unlikely that what it is like to see human faces is the same for infants and adults with early visual deprivation on the one hand, and adults with normal visual development on the other. The difference is clearly linked to perceptual organization: after long-term early visual deprivation subjects cannot learn to recognize individual faces or facial expressions. As LeGrand et al. (2001) showed, even a few months of visual deprivation in infancy cause subtle impairments in face discrimination ten years later. Thus ten-year-old children who were blind in the first 2-6 months of their life found faces with configural differences (distance between the eyes, nose, and mouth) more similar on average than control subjects. Therefore, perceptual integration explains a lot about the subjective aspect of experience, or so it seems. So why talk about qualia separately?

As we shall briefly argue, some types of phenomenal experience seem to occur independently of early perceptual learning. To make this point, first we shall focus on colour and luminance sensation and colour experience in infants, and subjects whose sight was restored after long-term blindness. Relevant data will be reviewed about the development of colour vision in infancy and childhood. Then some observations about vision in the newly sighted will be presented. Finally, a conclusion will be suggested, namely that overall, the development of colour perception relies, to a considerable extent, on genetically preprogrammed maturation, whereas the perception of form and space rely heavily on perceptual learning through childhood.

\section{Colour vision in children}

Infants at 2 months of age perform above chance in the forced-choice preferential looking (FPL) paradigm, when shown a red field embedded in a white surround. Despite varying the luminance of the red field (including the infant's brightness match), performance never dropped to chance level (Peeples and Teller, 1975; Teller, 1998). Using the same method for a wide range of chromatic stimulus fields, 2-month-olds succeeded for oranges, blue-greens and blues, but failed for yellow-greens and mid-purples (Teller et al., 1978). The majority of 1-month-old infants failed to make chromatic discriminations within this paradigm. The failures of 2-month-olds may suggest a tritan deficit at two months of age, however, in other studies two-month-old infants were able to make tritan discriminations (Teller, 1998, 3276). Red-green discriminations are mediated by the parvocellular pathway in adults, and probably also in infants (Dobkins et al., 1997; Bosworth and Dobkins, 2009) although some studies consider the possibility that magnocellular activity mediates certain responses to colour differences in infants (Dobkins et al., 1997). In these cases infants may detect changes in the colour of stimuli without experiencing differences in colour per se. The case in which this is most likely to happen is that of moving or counterphase flickering stimuli. Later studies suggested that the P-pathway may mature more slowly than the Mpathway (Dobkins and Teller, 1996; Dobkins et al., 1999; Bosworth and Dobkins, 2009, p8). As Teller (1998) summarizes these results, infants' responses to stationary, spatial chromatic

undergoing a phenomenal experience. Note also that even though your brain state particulars cannot occur in my brain, similar types of brain states may, and in this sense I may be able to share your experiences. 
changes are much more likely to reveal the presence of genuine colour experience in the infant's perceptual world. ${ }^{3}$

Illusions are invaluable tools for studying perception, since by means of investigating the 'errors' made by the visual system meticulously, we can draw conclusions on its working mechanisms. By studying differences in perception of illusions in different age-groups, answers to crucial developmental questions can be unveiled.

Yang et al.'s (2009) finding suggests that infant colour perception can be deceived similarly to that of adults, which implies that the mechanism behind colour perception must be well-developed at such an early age. In their study, 3-4-month-old infants showed evidence (preferential looking) of experiencing neon colour spreading illusion (FIG 1) in moving stimuli, but not in static ones. 5-6-month-olds on the other hand preferred neon colour spreading stimuli in both static and moving form. It is known that 4-month-old infants can perceive transparency in chromatic and moving patterns (Johnson and Aslin, 2000). It has also been demonstrated that the perception of transparency is necessary for the emergence of neon colour spreading (Meyer and Dougherty, 1987; Nakayama, Shimajo, and Ramachandran, 1990; Bressan, 1993a, 1993b). These data suggest that the perception of transparency and that of neon colour spreading develop together. Regarding our point, we think it is unlikely that infants' preference for stimuli inducing neon colour spreading does not arise from genuine colour experience - for instance that it arises from the activity of the luminance-sensitive Mcell pathway. That is, it seems that 5-6-month-old infants experience colours in ways comparable to that of older children and adults.

In addition to the neon colour spreading illusion, the Munker-White effect (FIG 2) was also studied by Yang et al. in another study (Yang et al, 2010). They showed that infants aged 4-8 months could perceive the illusion, that is, infants perceived pink targets more saturated if they were surrounded by more red than yellow. A looking preference paradigm was applied, based on the assumption that infants prefer high subjective saturation. In accordance with this expectation, infants showed significantly longer viewing times for the image in which the pink targets looked more saturated. However, the overall ratio of red in those images was inherently higher compared to yellow, whereas the images, whose pink targets looked less saturated, contained twice as much yellow as red. This entails that the images did not only differ in the perceived (illusory) saturation of the pink targets, but the overall physical saturation was higher in the images containing more red. Thus, in a second, control experiment, the authors applied the same colours but altered their spatial arrangement: the three colours of the original Munker-White configuration were shown as three large rectangles, dividing the image into three spatially uniform parts. In one of the two variants used, the red part covered half of the image, while it covered only its quarter in the other. Between these two figures, no significant difference in looking times was obtained; therefore, the authors concluded that the preference in the first experiment was due to the higher illusory saturation of the pink targets, rather than the higher overall ratio of red in those images. It can be noted, however, that in the second experiment, which was used as a control condition, children may have fixated exclusively the large uniform red squares, irrespective of their sizes, not scanning the image as a whole. This could also account for not obtaining significant difference in looking times in the control experiment. It would be worth applying an eyetracker in the control experiment of such studies, or to use a randomly mixed spatial arrangement of the colours while keeping their proportions constant. Nonetheless, to summarize the results reviewed so far, it seems that infants, as young as a few month of age,

\footnotetext{
${ }^{3}$ It is notable that tritan (blue-yellow) discriminations are mediated by a third retino-striatal pathway (the koniocellular one; Mollon, 2000), but this fact is rarely discussed in the infant colour vision literature where chromatic discriminations are most often equated with red-green discriminations.
} 
perceive colours in an adult-like manner, which is in line with our claim suggested in the present paper.

Another study that suggests that the perception of colour and luminance is genetically preprogrammed, or at least that it occurs particularly early in life, was conducted by Chien, Palmer and Teller (2005). This study compared the luminance perception of infants with that of adults. The authors examined whether infants' luminance perception deviated from Wallach's ratio rule in the same manner as the luminance perception of adults does. According to Wallach's (1948) ratio rule, the perceived lightness of a disk is determined by the ratio of the luminance of the disk and that of its surround. This rule provides a good approximation of human luminance perception, however, an approximately $10 \%$ deviation occurs in the direction of a luminance match. In a forced-choice novelty preference technique in combination with a cross familiarization paradigm, Chien et al (2005) found that children's luminance perception was more similar to that of adults, involving a certain deviation from Wallach's ratio rule, than to what Wallach's ratio rule would predict. In other words, infants deviated from Wallach's ratio rule in the same way as adults did. This result also supports the early and quick development of luminance perception.

However, there also exist data indicating that colour perception continues to improve later in childhood. Ling and Daina (2008) provide evidence that color discrimination measured by the Lanthohy New Color Test continues to improve up to 12 years of age. Whether this improvement is due to practice or preprogrammed maturation is not entirely clear - both factors may be relevant (see below for suggestion that preprogrammed maturation may play an important role). In addition, the literature is rich concerning the apparent difficulty that children between 2 and 5 years of age have with colour naming. This difficulty, however, stems from cognitive-linguistic factors, and not from children's ability to perceive colour (Sandhofer and Smith, 1999; Pitchford and Mullen, 2001; Fernald et al., 2010; Ramscar et al., 2007).

Thus colour perception - including phenomenal experience of the colours - occurs early in life. Does this imply that colour perception is genetically preprogrammed, or is this the manifestation of early perceptual learning? To investigate this question, we shall now review some case studies of the newly sighted.

\section{Colour vision in newly sighted patients}

Case studies of the newly sighted - people born blind and having their sight restored after an extended period of blindness - suggest that these subjects have severe difficulties with form perception and navigation in space, but their ability to perceive colour is much less affected. Discussion of these data must begin with a number of caveats, however. First, most of the existing evidence about the newly sighted is anecdotal, although the number of more precise studies has been growing recently. To our knowledge no study of colour vision in newly sighted subjects exists - that is, evidence on colour vision in the newly sighted is at present almost wholly anecdotal. Second, not all the reported cases are those of congenital blindness - a number of subjects had had good vision early on in their life before they became blind. Third, the majority of such subjects were not completely blind; they had residual lightness and colour sensations, sometimes even limited form perception. Fourth, in assessing colour vision in these subjects, two general aspects need to be examined: (i) their ability to experience colour in the phenomenal sense, (ii) the extent to which information about colour is integrated with other aspects of visual processing. Here we can only highlight some of the most relevant data concerning vision in the newly sighted before suggesting a conclusion.

A relevant case was described by Latta as early as 1904. Latta's patient was blind from birth and had only residual lightness sensation before his operation at age 30 (Latta, 1904). He 
learned to recognize colours quickly, starting with red and yellow, green taking a little longer to recognize. ${ }^{4}$ As Latta reports

\begin{abstract}
"The first tint that he saw was red. A red blanket lay across the foot of his bed. He asked what [color] it was, and was told; and never afterwards did he have the slightest hesitation in discriminating red again. ... He was shown a narcissus, and on being asked to describe it he immediately recognised the flower, and knew, from his old bouquet-making experience, that it was white and yellow; but he now for the first time also became aware of the little red band in the centre, and at once called attention to it." (Latta, 1904, 140)
\end{abstract}

Gregory and Wallace's patient S.B. performed similarly. 48 days after the operation he was familiar with yellows, greens, and blues, preferred the latter two, and complained about the large number of yellow shades. Colours stimulated him, and he used colours to recognize people by their clothes. Thus it seems that these patients have no problem with memory for colour. Chromatic resolution - hue discrimination - seems to be at normal levels. Here is what Ione Fine commented on her patient MM: "We did some crude tests for fine colour discrimination using Munsell chips and he was fine. Certainly nothing like the spatial acuity losses which were permanent. His isoluminant settings were also not noticeably abnormal" (Fine, electronic communication, July 2005). ${ }^{5}$ Colour also helps form perception. 48 days after his operation Gregory and Wallace's patient SB performed without a single error on the Ishihara tables - he did not even attempt to trace the digits with his finger. ${ }^{6}$ Based on a single observation, colour constancy appears to be incomplete after sight restoration. As I. Fine commented on MM: "Land like colour constancy was normal (compensating for colour filters over the eyes), but he could't interpret changes in colour due to shading - he basically didn't compensate for the change in colour due to shadows." (electronic communication, July, 2005).

Based on the available data (which is by no means abundant) it seems that after longterm (even congenital) blindness (i) colour experience appears virtually immediately, including normal levels of chromatic resolution (ii) recognition memory for colour develops quite fast, and is sufficiently stable, (iii) colour experience seems to be well integrated with what is available of form recognition and cross-modal perception. Some aspects of colour perception may still be affected - colour constancy is an example. Overall, these findings suggest that the development of colour perception may rely mainly on genetically preprogrammed maturation - more so than shape perception at least. However, thorough psychophysical studies of colour perception after long-term blindness are needed to secure this conclusion. It is also necessary to take a look at the development of the perception of form and space in order to conclude that these visual abilities require more perceptual learning than the vision of colour and luminance. Let us review such data on the newly sighted first, and then we will return to the investigation of the development of form and space perception (including binocular vision) in early and later childhood.

\footnotetext{
${ }^{4}$ Later this patient was suspected to be red-green colour deficient.

${ }^{5}$ Note that MM, Fine's patient was blind between ages 3 and 44. In this period he developed permanent amblyopia, but no severe deficit in colour discrimination.

${ }^{6} \mathrm{SB}$ was blind from 10 months to 53 years of age. His residual vision was insufficient to be of any use to him. He claimed to have had colour memories of red, white and black, but no other visual phenomena, from his blind period. Valvo's patient HS (blind between ages 15 and 37, had only lightness sensation during his blind period) underwent a colour vision test (Hertel tables). He had to trace the numbers on the tables to recognize them.
} 


\section{Perception of form and the three-dimensional world in newly sighted patients}

Newly sighted subjects quickly report the ability to detect motion and colour; they can also often recognize some forms like vehicles, or other objects that were familiar to them due to frequent tactile contact. Such forms are large capital letters or clock faces, which they learnt to read by touch at schools for the blind. Auditory stimulation also helped newly sighted patients to recognize outlines of human faces, knowing that speech sounds come from faces (Senden, 1960; Gregory and Wallace, 1963; Umezu et al., 1975; Sacks, 1995). Outlines of human forms against light were also recognized, however, form perception was not spontaneous, practice was necessary (Valvo, 1971). These data indicate that some degree of cross-modal perception must be immediately available. ${ }^{7}$ However, the same subjects had severe difficulty recognizing objects in photographs, different views of three-dimensional objects, and individual faces. Visual memory for shapes is a special challenge: sight-restored subjects keep forgetting, day after day, what they learn by sight about the shapes of familiar objects. They also find it surprising how objects "change their shape" as they walk around them (thus having difficulties with shape constancy).

Ambiguous depth illusions (Necker cube; staircase illusion) and perspective illusions are absent or greatly reduced (Gregory and Wallace, 1963). Visual acuity is substantially reduced, and there is rather limited improvement in the years following surgery (Fine et al., 2003; Umezu et al., 1975). In a recent study, contour integration (using short line segments) was not far from control levels; the perception of Glass patterns was good too. On the other hand, illusory contours in Kanizsa figures were not seen (Fine et al., 2003; for psychophysical tests of another recovery case see Fine et al., 2002).

This traditional picture has been refined recently (Maurer et al., 2005; Ostrovsky et al., 2009). Some phenomena of early deprivation are explained by Hebbian competition - pruning of exuberant neural connections that are present in early infancy. One such example is suppression of the non-functional eye by the active one in monocular deprivation. Still, a number of phenomena need different explanations. Recent studies indicate that visual input is necessary for maintaining normal visual function even after the age when performance reaches adult levels. For instance, short periods (even a couple of weeks) of visual deprivation before age 10 cause permanent deficits in letter acuity (which reaches adult levels by age 6). However, not all visual connections need input for maintenance. Sensitivity to global motion (tested by dot patterns) is one example. Perception of global motion relies on area V5. Global motion sensitivity (including corresponding V5 activity) is normal even when deprivation begins at 4 months of age - despite the fact that visually normal 4-month-olds are seven times less sensitive to the global direction of motion than adults. In addition, for some visual functions, there is plasticity even in adolescence and adulthood. In some cases deprivationinduced amblyopia was seen to dramatically improve as a result of patching the other eye for 6-12 months between ages 7-10, or when vision in the fellow good eye was lost. These observations indicate that some exuberant neural connections are inhibited rather than pruned. There are interesting differences between the detection of global motion and global form (as in Glass patterns). It seems that there is a critical period for the detection of global motion in the first four months of life. Blindness onset after this age does not seem to affect the detection of global motion (and the corresponding activity in area V5) whereas congenital blindness does cause a severe deficit in global motion threshold. Regarding global form, after the removal of bilateral or unilateral congenital cataract both monocular and binocular

\footnotetext{
${ }^{7}$ Thus the data suggest a partial positive answer to Molyneux's question: Irish scientist William Molyneux in 1688 sent a letter to English philosopher John Locke asking whether a congenitally blind subject familiar with a cube and a sphere through touch could distinguish them by vision alone, if their vision had been restored (see Morgan, 1977; Evans, 2002).
} 
thresholds were above normal levels, but the difference was smaller than that for global motion. Maurer et al., suggest three points. First, early experience can be important in preserving the infrastructure for later learning, without that experience inducing any learning at the earlier time. Second, in certain cases there is greater deficit for the aspect of vision that matures slower in visually normal children. However, there are also exceptions to this principle. Third, early deprivation affects both the ventral and the dorsal system of vision, and there is no obvious overall difference in plasticity between the two streams.

Ostrovsky et al. (2009) examined high-level form processing in three subjects shortly after recovery. They found that static cues for image parsing like good continuation and junction structure were ineffective. The subjects oversegmented partially overlapping geometrical figures (e.g., an overlapping triangle and circle were identified as three objects), and had difficulty recognizing photographs of real-world objects. Their form processing appeared to rely on low-level attributes like segmenting along patches of homogeneous luminance and hue. However, making the imaged objects move helped grouping together static cues that belonged to the same object; motion cues greatly supported correct segmentations and object recognition. In addition, over the course of 10 to 18 months, all three subjects improved on the tasks; they became better at parsing static images.

Thus, it seems that hue and colour information is readily available, while high-level form processing can gradually improve even after blindness extending into adulthood, and the improvement can occur on the basis of daily activities, without any special training.

\section{Development of form and visual context perception}

Though many crucial visual functions are shown to emerge during the first year of life, visual development is not finished until the end of childhood (Kovács, 2000). There is an observable inhomogeneity in the development of different visual functions and the maturation of neuroanatomical circuits participating in visual information processing. In contrast with the data that suggest that colour vision develops early in life, integration of contours (Kovács, 2000) or of the visual context (Káldy and Kovács, 2003) is not fully developed even at the age of 4-5 years.

Káldy and Kovács (2003) examined the effect of visual context on size perception by means of the Ebbinghaus illusion (or Titchener circles, FIG. 3). They compared the magnitude of the Ebbinghaus illusion in 4-year-old children and adults. Applying a 2AFC design, in which the subjects had to judge which of the two presented disks was bigger, they found that children were significantly less deceived by the illusion than adults, and perceived the target disk surrounded by disks of different sizes in accordance with its veridical size. The perception of adults was more misled by the context. The authors suggest that the reduced contextual sensitivity in children is due to immature cortical connectivity.

Hanish, Konczak and Dohle (2001) also used the Ebbinghaus illusion to investigate the effect of spatial context in older age groups, between the ages of 5 and 12 years. The perceptual judgement task was to judge whether the two disks presented in the contexts of two different sizes were of the same or different size. They randomly exchanged the target disks to larger and smaller ones, repeating each 3 times, while keeping the size of the reference disk constant. They report that when pure perceptual judgements had to be made, children were deceived by the illusion to the same extent as adults, i.e. they produced the same size estimations, which might imply that maturation is gradual, and although 4 year-olds are not deceived by the context (Káldy and Kovács, 2003), neural connectivity is getting closer to the 
adult level by 5-12 years of age. On the other hand, in Hanish et al's study, when subjects were presented again with the configuration using the same disk sizes they had previously judged as equal, , adults were deceived significantly more $(81 \%)$ than the two groups of children (55\% for 5-7 year-olds and 63\% for 8-12 year-olds).

In Duemmler et al's (2008) study, a single configuration was shown, contrary to other studies mentioned here. In Duemmler et al' s perceptual task, an isolated comparison disk was presented to the left or to the right of the single illusion context, and the participant's task was to judge whether the comparison disk was smaller or larger than the target disk in the centre of the illusion context. In this study, different results were obtained compared to the studies mentioned previously: they found a stronger illusion for the Ebbinghaus configuration in 5-7 year-olds than in 8-12 year-olds. As this study did not include an adult control group, it offers no direct comparison of children's data with that of adults.

Another illusion that involves the integration of spatial context in the perception of size is the Müller-Lyer illusion (FIG. 4). Rival and her co-workers (2003) investigated the Müller-Lyer illusion in children aged 7,9 and 11 years. The task of the subjects was to select the bar from the 5 bars of different sizes, whose size matched that of the target bar, presented in the illusion context. Their perceptual task results imply that the illusion was present in children, though 11 year-olds were more deceived than 7-year-olds. All the three age groups underestimated the size of the line with outward-pointing arrows at its end, but the inwardpointing arrows made only the 11 year-olds overestimate line size. Unfortunately, this study did not include an adult control group either.

Kovács et al (1999) investigated the effect of noise on contour integration in the age range 5-35 years. Their stimuli consisted of open and closed contours (circles or ovals) of Gabor signals, embedded in noise (randomly distributed Gabor signals) of varying density. They found a significant improvement in contour integration between 5 and 14 years of age. They also tested whether learning was specific for the stimulus dimensions of orientation and colour in the contour-detection task, by testing whether there was a transfer of learning across these cues. Therefore, colour cards were also used, in which the contour to be detected comprised elements of the same colour, and noise of different colours was also added to these cards, so that the task difficulty matched the contour card condition. The findings in these tasks suggest that this result is an indication of low-level perceptual development, since though performance of their subjects improved with practice in both conditions; this improvement was cue-specific, since no transfer occurred.

\section{Development of binocular vision}

An interesting way to study binocular vision is to present two different images to the two eyes (FIG 5). Such image pairs cannot be fused into a single 3D percept; instead, binocular rivalry occurs. In binocular rivalry, the visual system vacillates between the two percepts spontaneously, producing either a clear percept of one or the other image, or a patchwork of the two. This phenomenon is due to the fact that two different objects cannot occupy the same location in real 3D scenes (Kovács, Papathomas, Yang and Fehér, 1996). Therefore it is plausible to suppose that binocular rivalry occurs in ontogenesis only when 
binocular fusion has also developed. Nonetheless, there is very little research on the development of binocular rivalry in childhood.

The earliest age at which the presence of stereopsis was shown, is 3 weeks (Mocan, Wright and Salvador, 2007). Aslin and Dumais (1980), however, list a number of constraints that prevent infant binocular functions from being adultlike, such as acuity and contrast sensitivity, accommodation, and facial dimensions. Infants younger than 3 months of age might also have a difficulty in keeping stable bifoveal fixation (Aslin and Dumais, 1980).

Whether there is a critical period in human development for binocular vision, and what is perceived by infants before the occurrence of binocular 3D perception are debated issues. The existence of a critical period in the development of binocular vision in the cat has long been known (Hubel and Wiesel, 1965). As for humans, children who suffer deprivation of binocular input in the first 3 years of their lives, never develop normal binocular function, even when their eyes are aligned by surgery. Sensitivity to binocular deprivation can be found up to the age of 9 years (Aslin and Dumais, 1980). However, there also exist data that support plasticity after the supposed critical period, implying that proper treatment can reassemble binocular functions (Blake and Wilson, 2011).

The nature of binocular vision in human infants before the occurrence of binocular 3D perception is also debated. This issue was mainly investigated by means of preferential looking paradigms using stimuli that induce binocularly rivalry in adults. Shimojo, Bauer, O'Connel and Held (1986) found that infants younger than 3.5 months of age preferred to look at the dichoptic (interocularly orthogonal) pattern. However, at the age of 3.5 months on average, a sudden shift of preference occurred from the rivalrious pattern to the fusible stimulus. The authors interpret this result as a preference for a blended stimulus, resulting in a grid-like pattern, which is more complex than the monocularly projected simple lines. However, once binocular functions have developed (around 3,5 months of age), the two patterns begin to oscillate, which is aversive for infants. This would account for the shift in the preference for binocularly fusible stimuli. On this basis, pre-stereoscopic vision seems to blend images that are rivalrous for adults. However, attempts to replicate these results were unsuccessful (Brown and Miracle, 2003). Neither did Brown, Candy and Norcia (1999) find any physiological evidence for binocular rivalry using a visually evoked potential paradigm with 5-15 months old infants. They attribute their result to the immaturity of dichoptic suppression.

Even less data has accumulated so far concerning the development of binocular vision and rivalry following infancy. In a study which aimed to compare binocular interactions of children aged 6-14 years to normal and amblyopic adults, it was found that binocular summation decreased with age in a dichoptic visual acuity task compared to its monocular counterpart (Vedamurthy, Suttle, Alexander and Asper, 2007). The acuity of the dominant eye did not improve significantly in children in the dichoptic viewing condition compared to the monocular condition. In this respect, the performance of children was similar to that of adults. However, they found a significant negative correlation with age in the improvement of acuity of the non-dominant eye in the dichoptic condition compared to the monocular one. The difference between the groups of children and adults was not significant, which might be due to the wide age range of children examined. These preliminary results therefore indicate 
that development in binocular interactions continues after infancy, until at least pre-puberty. Our recent study also supports this claim (Hudák, Gerván, Friedrich, Pastukhov, Braun and Kovács, 2011). Our data indicate that even pre-puberty children tend to alternate more quickly than adults, in which a larger extent of adaptation might play a significant role.

The development of binocular rivalry was investigated in 5 to 6 year-old children (Kovács and Eisenberg, 2005). Their results also indicated that children alternated significantly more quickly than adults. Based on this finding, the authors suggest that children must have perceived patchworks of the two images more frequently than did adults. They argue that the visual system of 4-5-year-old children is not sufficiently mature to integrate entire images spatially, thus they see more piecemeal rivalry than adults. This is in line with their earlier findings in contour integration (Kovács, 2000) or spatial integration (Káldy and Kovács, 2003).

Some data has also been collected concerning the changes in binocular rivalry during adulthood (Jalavisto, 1964; Ukai et al. 2003). The main finding of these studies is that domination times became longer with age. Jalavisto (1964) focused on binocular oscillations in the age range of 40-93. The result of this study was that the frequency of oscillation decreased with age in a regular manner, and a total lack of change became prevalent in the oldest age classes. The results of a more recent study are in line with these findings (Ukai et al., 2003), in which the alternation rates in three age-groups were compared: 20-34, 35-49, and 50-64-year-old subjects were investigated. They obtained similar results to that of Jalavisto (1964): a prolongation in alternation time as a function of age was found, which is also in line with the developmental studies reviewed so far.

\section{Other forms of bi-stable perception}

Another perceptual phenomenon that is related to binocular rivalry is the perception of ambiguous figures. Although binocular rivalry is the consequence of the failure to match the two different images with the same spatial position in the 3D world (Kovács et al. 1996), it shares several features with the alternation of one or the other interpretation of ambiguous figures, such as the Necker-cube or the duck-rabbit figure (FIG. 6). Such common features are the gamma distribution of the dominance times of each percept, the high inter-subject variability of the frequency of reversals, the significant influence of stimulus properties on reversal rates, and the fact that both can be influenced by the voluntary control of the subject (see Kornmeier and Bach, 2005).

According to the findings reviewed by Leopold and Logothetis (1999), stimulus properties, such as brightness, contrast and spatial-frequency content can have a significant impact on the balance of dominance and suppression. In addition, high-level properties of the stimuli can also modify dominance periods in multi-stable perception. Such properties include recognisability or semantic content. For instance, if a recognizable figure is inverted upside down, then its perceptual dominance might significantly be altered in both figure/ground stimuli and binocular rivalry.

Voluntary control is another factor affecting multi-stable perception. Subjects' voluntary control had a stronger influence on the perception of ambiguous figures than that of binocularly presented rivalrous stimuli (van Ee et al, 2005). Taddei-Ferratti et al (2008) also 
point out that the rivalry between the two possible percepts of an ambiguous figure is less automatic than the competition between two different images presented binocularly. An additional common feature of binocular rivalry and ambiguous figures that Taddei-Ferratti et al (2008) mention is that both are influenced by eye-movements (Ellis and Stark, 1978; Sabrin and Kertesz,1980).

Reese and Ford's (1962) pioneering study intended to investigate developmental aspects of ambiguous figure perception. Nursery-school children were shown a series of six pictures of either animals or human faces. Their task was to name each. After that, they were asked to state their expectancy about the next picture. The result was that when they were shown the Bugelski rat-man ambiguous figure, it was easier for them to provide the "animal" interpretation than the "human face" interpretation, which means that the animal interpretation was easier to prime by the previously shown pictures. This might be considered evidence of stimulus-dependency even at such an early age. In addition, there are significant performance differences as well, within the age range from 3 to 5 years. Doherty and Wimmer (2005) found that 3-year-old children cannot even report both interpretations of such ambiguous images as the duck-rabbit or the man-mouse figures. However, 4-year-old children can easily interpret the ambiguous figures in both ways. Nonetheless, spontaneous reversals occurred only at the age of 5. The conclusion of this study is that understanding that the perception of the same physical image might reverse is not sufficient for spontaneous reversals to occur.

The foregoing review indicates that so far there has been relatively little research concerning binocular vision and bistable perception in childhood. No data can be found on the later development of binocular integration and rivalry for the ages following 5-6 years, and there is little data even for earlier periods. Research on form and spatial integration development is also scarce, therefore on the basis of the available data, we cannot draw a comprehensive picture of visual development.

\section{Conclusions}

The emerging view seems to be that concerning luminance-and colour perception, the perceptual world of children might be very similar to that of adults, even such early ages as a few months. Colour and luminance discrimination and perception seem to work in an adultlike manner in children, and this is also the case with newly sighted patients. Regarding illusory color experiences, small children and adults exhibit important similarities; however, there is a shortage of data concerning the sight restored.

In contrast, at higher levels of perception, such as binocular vision or the interpretation of ambiguous figures, as well as the integration of spatial information present in the entire visual field, longer periods of maturation and learning are necessary in order to attain the perceptual abilities and therefore, the phenomenology experienced by normal-sighted healthy adults. This maturation continues up to at least pre-puberty, and plasticity for such perceptual skills can also be shown in adult subjects.

Therefore, based on the reviewed data, the conclusion can be drawn that the development of colour perception involves a genetically preprogrammed maturation to a considerable extent. In contrast, the perception of form and space as well as binocular vision and perception of ambiguity of forms rely heavily on perceptual learning through childhood, 
and everyday perceptual experience is also crucial for the development of form and space perception of newly-sighted adults.

\section{Figure legends}

Figure 1. Neon colour spreading illusion. The areas around the red crosses of the intersections are physically uniform white. However, a reddish veiling disk spreading in a neon-like manner is perceived around the red crosses.

Figure 2. Munker-White illusion. The two rows of pink rectangles are physically of the same colour. However, the lower row of pink rectangles, that are surrounded by more red than yellow, look more saturated than the upper row.

Figure 3. Ebbinghaus illusion. The middle disks of the two configurations are of the same physical size. However, the disk on the left surrounded by smaller disks looks noticeably larger than the one in the right, surrounded by large disks.

Figure 4. Müller-Lyer illusion. The length of the two vertical lines are identical. However, the one on the left, ending in an outward-pointing arrow, looks much shorter than the one on the right, ending in an inward-pointing arrow.

Figure 5. Binocular rivalry (after Hudák et al., 2011). The red and the green gratings should be viewed through red-green anaglif glasses, stimulating the two eyes separately with the two gratings. In this case, the perception of the two gratings is alternating spontaneously, or the mixture of the two gratings is perceived.

Figure 6. Duck-rabbit ambiguous figure. The drawing can be perceived either as a duck or a rabbit, but the two interpretations cannot be present at one time. Rotation of the image can help switching. The left and the right images are physically identical, but the left image is rotated 10 degrees clockwise, and is easier to perceive it as a rabbit, whereas the right image is rotated 15 degrees counter-clockwise, triggering the duck interpretation more easily. The two interpretations can still be alternating spontaneously in both views, though.

\section{References}

Aslin, R.N., and Dumais, S. T (1980). "Binocular Vision in Infants: A Review and a Theoretical Framework" in Advances in child development and behaviour, ed. Reese, H. W., and Lipsitt, L. P. (New York, USA: Academic Press Inc.), 53-94.

Blake, R., and Wilson, H. (2011). Binocular vision. Vis. Res. 51, 754-770.

Bosworth, R., G., Dobkins, K., R. (2009). Chromatic and luminance contrast sensitivity in fullterm and preterm infants. Journal of Vision, 9 (13): 15, 1-16

Bressan, P. (1993a). Neon colour spreading with and without its figural prerequisites. Perception, 22, 353-361. 
Bressan, P. (1993b). Revisitation of the luminance conditions for the occurrence of the achromatic neon colour spreading illusion. Perception \& Psychophysics, 54, 55-64.

Brown, R. J., Candy, T. R., and Norcia, A. M. (1999). Development of rivalry and dichoptic masking in human infants. Investigative Ophthalmology and Visual Science 40, 3324 3333.

Brown, A. M., and Miracle, J. A. (2003) Early binocular vision in human infants: limitations. Vis. Res. 43, 1563-1574.

Dobkins, K., R., Sampath, V. (2008). The use of chromatic information for motion segmentation: Differences between psychophysical and eye-movement measures. Perception, 37, 993-1009.

Dobkins, K., R., Teller, D., Y. (1996). Infant Motion:Detection (M:D) Ratios for Chromatically Defined and Luminance-defined Moving Stimuli, Vision Research, 36 (20) 3293-3310.

Dobkins, K., R., Lia, B., Teller, D., Y. (1997). Infact Colour Vision: Temporal Contrast Sensitivity Functions for Chromatic (Red-Green) Stimuli in 3-Month-olds. Vision Research, 37 (19) 2699-2716.

Dobkins, K., R., Anderson, C., M., Lia, B. (1999). Infant temporal contrast sensitivity functions (tCSFs) mature earlier for luminance than for chromatic stimuli: evidence for precocious magnocellular development? Vision Research, 39 3223-3239

Doherty, M. J. and Wimmer, M. C. (2005). Children's understanding of ambiguous figures: Which cognitive developments are necessary to experience reversal? Cognitive Development, 20, 407-421.

Evans, G. (2002). Molyneux's question. In Alva Noe and Evan Thompson (eds). Vision and Mind. Cambridge Mass. MIT Press

Fernald, A., Thorpe, K., Marchman, V., A. (2010). Blue car, red car: Developing efficiency in online interpretation of adjective-noun phrases, Cognitive Psychology, 60, 190-217.

Fine, I., Smallman, H., S., Doyle, P., MacLeod, D., I., A. (2002). Visual function before and after the removal of congenital cataracts in adulthood. Vision Research, 42, 191-210.

Fine, I., Wade, A., Brewer, A., A., May, M., G., Goodman, D., F., Boynton, G., M., Wandell, B., A., MacLeod, D., I., A. (2003). Long-term deprivation affects visual perception and cortex. Nature Neuroscience, 6 (9) 915-6

Gopnik, A., and Rosati, A. (2001). Duck or rabbit? Reversing ambiguous figures and understanding ambiguous representations. Developmental Sci. 4, 175-183.

Gregory, R., L., Wallace J., G. (1963). Recovery from Early Blindness; A Case Study. Experimental Psychology Society Monograph No. 2. Available at: http://www.richardgregory.org/papers/recovery_blind/contents.htm

Hayne W. Reese, LeRoy H. Ford Jr (1962). Expectancy and perception of an ambiguous figure in preschool children. J. of Verbal Learning and Verbal Behavior, 1( 3), 188191.

Hubel, D. H., and Wiesel, T. N. (1965). Binocular interaction in striate cortex of kittens reared with artificial squint. J. of Neurophysiol., 28, 1041-1059. 
Hudák M, Gerván P, Friedrich B, Pastukhov A, Braun J and Kovács I (2011) Increased readiness for adaptation and faster alternation rates under binocular rivalry in children. Front. Hum. Neurosci. 5:128. doi: 10.3389/fnhum.2011.00128

Jalavisto, E. (1964). The phenomenon of retinal rivalry in the aged. Gerontologia 9, 1-8.

Johnson, S., Aslin, R. (2000). Infants' perception of transparency. Developmental Psychology, 36, 808-816.

Káldy, Zs., and Kovács, I. (2003). Visual context integration is not fully developed in 4-yearold children. Perception, 32, 657 - 666.

Kornmeier, J. and Bach, M. (2005). The Necker cube-an ambiguous figure disambiguated in early visual processing. Vis. Res., 45, 955-960.

Kovács, I. (2000). Human development of perceptual organization. Vis.Res., 40, 1301-1310.

Kovács, I. and Eisenberg, M. (2005). "'Human development of binocular rivalry" in Binocular rivalry, ed. Alais, D. and Blake, R. (Cambridge, Massachusetts, London, England: MIT Press). 101-116.

Kovács, I., Papathomas, T. V., Yang, M., and Fehér, Á. (1996). When the brain changes its mind: Interocular grouping during binocular rivalry. Proc. Natl. Acad. Sci., 93, 1550815511.

Latta, R. (1904). Notes on a Case of Successful Operation for Congenital Cataract in an adult. British Journal of Psychology 1, 135-150.

LeGrand, R., Mondloch, C., J., Maurer, D., Brent, H., P. (2001). Early visual experience and face processing. Nature, $410,890$.

Ling, B., Y., Daina, S., J. (2008). Colour vision in children and the Lanthony New Colour Test, Visual Neuroscience, 25, 441-444

Maurer, D., Lewis, T., L., Mondloch, C., J. (2005).Missing sights: consequences for visual cognitive development. Trends in Cognitive Sciences, 9 (3) 144-151.

Meyer, G., Dougherty, T. (1987). Effects of flicker-induced depth on chromatic subjective contours. Journal of Experimental Psychology: Human Perception and Performance, 13, 353-360.

Mocan, M. C., Wright, K. W., and Salvador, M. G. (2007). Evidence of binocular fusion in a 3-week-old infant with transient abducens nerve paresis. J. of American Association for Pediatric Ophthalmology and Strabismus, 11(2), 199-200.

Mollon, J. (2000): "Cherries among the Leaves": The Evolutionary Origins of Colour Vision. In Steven Davis (ed.) Colour Perception: Philosophical, Psychological, Artistic and Computational Perspectives. Oxford University Press, Oxford

Morgan, M.J., 1977, Molyneux's Question: Vision, Touch and the Philosophy of Perception, Cambridge: Cambridge University Press.

Nakayama, K., Shimajo, S., Ramachandran, V., S. (1990). Transparency: Relation to depth, subjective contours, luminance, and neon colour spreading. Perception, 19, 497-513.

Ostrovsky, Y., Meyers, E., Ganesh, S., Mathur, U., Sinha, P. (2009). Visual Parsing After Recovery From Blindness, Psychological Science, 20, (12), 1484-1491.

Teller, D., Y. (1998). Spatial and temporal aspects of infant colour vision. Vision Research 38, 3275-3282. 
Teller, D., Y., Peeples, D., R., Sekel, M. (1978). Discrimination of chromatic from white light by two-month-old human infants. Vision Research, 18(1), 41-48.

Tye, M. (2000). Consciousness, Colour, and Content. Cambridge, MA: MIT Press

Peeples, R., R., Teller, D., Y. (1975). Colour viison and brightness discriminationin twomonth-old human infants. Science, 189, 1102-3.

Pitchford, N., J., Mullen, K., T. (2001). Conceptualization of Perceptual Attributes: A Special Case for Colour? Journal of Experimental Child Psychology, 80, 289-314.

Ramscar, M., Thorpe, K., Denny, K. (2007). Surprise in the Learning of Colour Words. Proceedings of the 29th Meeting of the Cognitive Science Society, Nashville, TN.

Sacks, O., (1995). To See and Not see. In O. Sacks: An anthropologist on Mars, Picador

Sandhofer, C., M., Smith L., B. (1999). Learning Colour Words Involves Learning a System of Mappings, Developmental Psychology, 35 (3), 668-679

Senden, Max von (1960). Space and Sight: The Perception of Space and Shape in the Congenitally Blind Before and After Operation. London: Methuen \& Co. Ltd.

Shimojo, S., Bauer Jr, J., O'Connell, K.M., and Held, R. (1986). Pre-stereoptic binocular vision in infants. Vis. Res., 26(3), 501-510.

Ukai, K., Ando, H., and Kuze, J. (2003). Binocular rivalry alternation rate declines with age. Percept. Mot. Skills 97, 393-397.

Umezu, H., Toril, S., Uemura, Y. (1975). Postoperative formation of visual perception in the early blind. Psychologia, 18, 171-186.

Valvo, A. (1971). Sight restoration after long-term blindness: The problems and behavior patterns of visual rehabilitation. New York: American Foundation for the Blind

Vedamurthy, I., Suttle, C. M., Alexander, J. and Asper, R. J. (2007). Interocular interactions during acuity measurement in children and adults, and in adults with amblyopia. Vis. Res., 47, 179-188.

Yang, J., Kanazawa, S., Yamaguchi M., K. (2009). Percetion of neon colour spreading on 36-month-old infants. Infant behavior \& Development, 32, 461-467. 